\title{
Conductive Polymer Composites Fabricated by Disposable Face Masks and Multi-Walled Carbon Nanotubes: Crystalline Structure and Enhancement Effect
}

\author{
Meng Xiang ${ }^{1}$, Zhou Yang ${ }^{1}$, Jingjing Yang ${ }^{1}$, Tong Lu ${ }^{1}$, Danqi Wu ${ }^{1}$, Zhijun Liu ${ }^{1}$, Rongjie Xue ${ }^{1}$ and \\ Shuang Dong ${ }^{2 *}$ \\ ${ }^{1}$ Department of Materials Engineering, Jiangsu University of Technology, Changzhou, 213001, China \\ ${ }^{2}$ Department of Mathematics Science and Chemical Engineering, Changzhou Institute of Technology, Changzhou, 213032, China \\ *Corresponding Author: Shuang Dong. Email: dongs@czu.cn
}

Received: 03 May 2021 Accepted: 15 June 2021

\begin{abstract}
Influenced by recent COVID-19, wearing face masks to block the spread of the epidemic has become the simplest and most effective way. However, after the people wear masks, thousands of tons of medical waste by used disposable masks will be generated every day in the world, causing great pressure on the environment. Herein, conductive polymer composites are fabricated by simple melt blending of mask fragments (mask polypropylene, short for $\mathrm{mPP}$ ) and multi-walled carbon nanotubes (MWNTs). MWNTs were used as modifiers for composites because of their high strength and high conductivity. The crystalline structure, mechanical, electrical and thermal enhancement effect of the composites were investigated. MWNTs with high thermal stability acted the role of promoting the crystallisation of $\mathrm{mPP}$ by expediting the crystalline nucleation, leading to the improvement of amount for crystalline nucleus. MWNTs fibers interpenetrate with each other in mPP matrix to form conducting network. With $2.0 \mathrm{wt} \%$ MWNTs loading, the tensile strength and electrical conductivity of the composites were increased by $809 \%$ and 7 orders of magnitude. MWNTs fibers interpenetrate with each other in mPP matrix to form conducting network. Thus, more conducting paths were constructed to transport carriers. The findings may open a way for high value utilization of the disposable masks.
\end{abstract}

\section{KEYWORDS}

Disposable face masks; multi-walled carbon nanotubes; crystalline structure; mechanical enhancement effect; conducting network

\section{Introduction}

Influenced by recent COVID-19, wearing face masks to block the spread of the epidemic has become the simplest and most effective way [1]. However, after the people wear masks, there are also some inevitable but not enough attention to follow-up problems, among which the most important is how to deal with the used disposable masks. If the average weight of a common three-layer disposable mask is $5 \mathrm{~g}$, thousands of tons of medical waste will be generated every day in the world, causing great pressure on the environment [2]. The structure of the common disposable mask is divided into three parts: the mask body, the ear straps and nose seals. The mask body is a non-woven fabric composed of high fluidity polypropylene (PP) resin, the ear straps were elastomer composed of polyurethane, and the nose seals were composed of iron wire and 
polypropylene resin. In addition to the fact that the nose seals are difficult to be reused because of the iron wire, polymer composites with high performance and high added value could be easily prepared by using the structural characteristics of the rest parts of the disposable mask, which has good ecological and economic benefits, and is helpful to solve the problems of environmental pollution and resource shortage in our society.

For the primitive polymer materials, the surface resistivity range is within $10^{12}-10^{14} \Omega$, which easily leads to the build-up of static charge on the surface of polymer materials, as well as the dust contamination, short circuit and even cause a fire or an explosion in explosive, mining equipment, packaging of electrical devices and so on [3]. Therefore, conductive composites with improved antistatic property were required in those areas [4]. Compared with conductive metal composites, conductive polymer composites have lower density, better flexibility, simpler preparation and lower cost [5]. However, traditional conductive polymer composites are usually made of raw materials from petrochemical resources, which consume a lot of non-renewable petrochemical resources. If the raw material used in the study was neat PP, the increase of tensile strength of the composites was limited [6]. High molecular weight of neat PP leads to high melt viscosity in the preparation of the composites, in which the MWNTs would be more difficult to uniformly dispersed in neat PP matrix, leading to a poor increase of mechanical property of the composites. On the other hand, compared with neat PP, the molecular weight of the recycled PP decreases due to thermal oxidative degradation, leading to the lower melt viscosity in the preparation of the composites, in which the MWNTs would be easier to uniformly dispersed in recycled PP matrix, leading to a higher increase of mechanical property of the composites. At present, researchers have made some progress in this field of high value utilization of recycled PP and recycled fillers: Belviso et al. [7] investigated a rapid recycling method of waste polypropylene (WPP) from automotive industry with the addition of recycled inorganic fillers. The results indicate that the addition of low percentage $(5.0 \mathrm{wt} \%)$ of sepiolite or zeolite improves tensile modulus of WPP-recycled materials by 5.4\%. Ajorloo et al. [8] incorporated of recycled PP and wasted fly ash in PP-based composites for automotive applications. The impact strength of the recycled composites increased by $23.3 \%$ under $30.0 \mathrm{wt} \%$ fly ash. Bahri et al. [9] fabricated functionally graded natural filler-recycled polypropylene (FGNF-RPP) composite and investigated deflection behaviour under static mechanical loads. The results showed that composite under $40.0 \mathrm{wt} \%$ waste wood sawdust produced the least deflection. Barczewski et al. [10] produced polymer composites based on recycled PP incorporating ground sunflower husk. The elasticity modulus of the green composites was increased by $19.4 \%$ in the filler amount of $20.0 \mathrm{wt} \%$. Battegazzore et al. [11] proposed a mechanical recycling protocol of disposable masks by directly melt blending the mask body with ear straps. However, The Young's Modulus and maximum stress of the composites were decreased with the ear straps loading compared with only use mask body.

The focus of recent researches has been mostly on the effect of reprocessing on the mechanical properties of recycled commercial PP and different fillers. However, very few publications are available in the literature that discusses the electrically or thermally conductive properties of recycled PP from disposable face masks. In this research, conductive polymer composites are fabricated by simple melt blending of mask fragments (mask polypropylene, short for mPP) and multi-walled carbon nanotubes (MWNTs). At our processing temperature of $180^{\circ} \mathrm{C}$, the COVID-19 virus will be quickly inactivated [12]. MWNTs were used as modifiers for composites because of their high strength and high conductivity. The crystalline structure, mechanical, electrical and thermal enhancement effect of the composites were investigated. To the best of the authors' knowledge, this is the first study investigating the electrically and thermally conductive properties of the green composites from disposable mask fragments. The obtained composites could be applied to the fields of sustainable railway sleepers [13], 3D printing feedstock materials [14] and low value building materials [15]. The importance of this research gets highlighted when we consider that the reuse of disposable mask fragments may be an 
environmental and economic solution, which could help to the efficient elimination of waste materials from the biosphere to some extent.

\section{Experimental}

\subsection{Materials}

The disposable mask (Henan Chaoya) and MWNTs (Huizhou Tannatong) were purchased. After removed nose seals and ear straps, the disposable mask was smashed into mask fragments (mask polypropylene, short for $\mathrm{mPP}$ ).

\subsection{Preparation}

Firstly, the polypropylene fabric was directly compressed at $180^{\circ} \mathrm{C}$ for $5 \mathrm{~min}$ after removing nose seals and ear straps, as shown in Fig. 1a. After that, we cut the compressed products into small pieces with scissors, as shown in Fig. 1b. Finally, we added the small pieces of polypropylene fabric (mPP) in to the internal mixer at $180^{\circ} \mathrm{C}$ for $5 \mathrm{~min}$ again for the preparation of the $\mathrm{mPP} / \mathrm{MWNTs}$ composites (Fig. 1c).
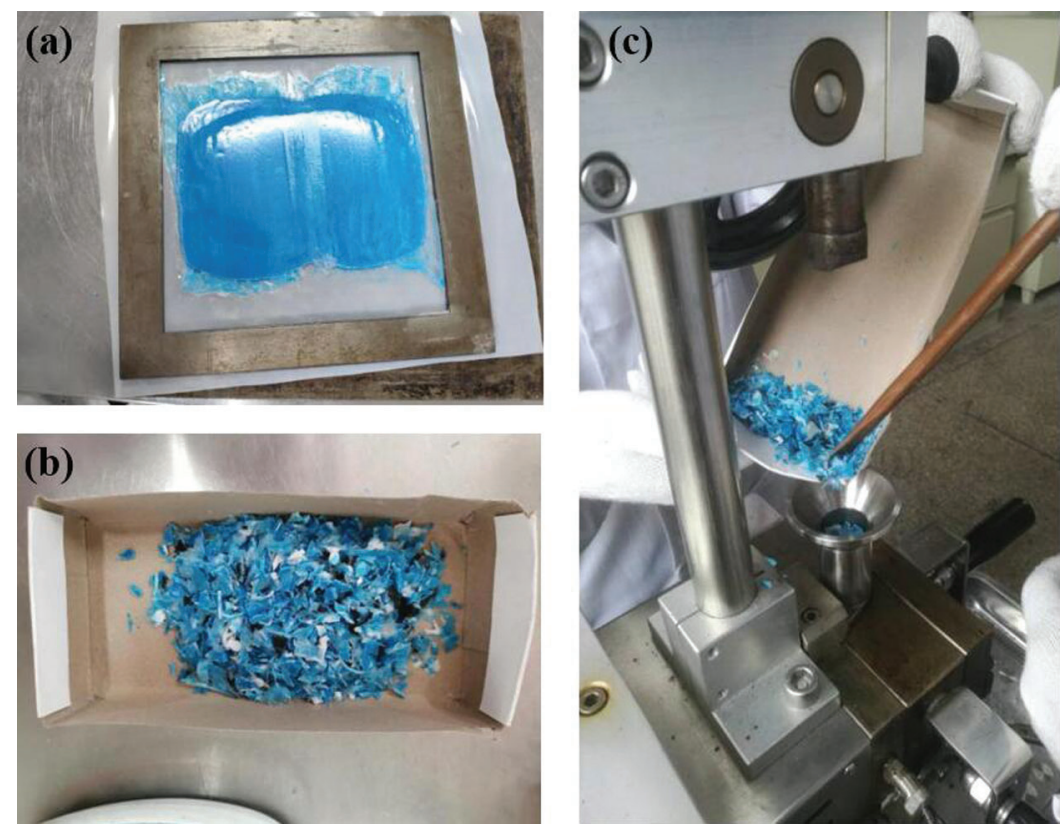

Figure 1: Compressing (a), cutting (b) and feeding (c) of mPP

The $\mathrm{mPP}$ were mixed with MWNTs at $180^{\circ} \mathrm{C}$ for $5 \mathrm{~min}$. After that, the composites were prepared by compressing the mixed products at $180^{\circ} \mathrm{C}$ for $5 \mathrm{~min}$ again (Fig. 2). The samples were named as $\mathrm{mPP} / \mathrm{Xwt} \% \mathrm{MWNTs}$, in which X denotes MWNTs content in the composites.

\subsection{Measurements}

The Fourier-transform infrared (FT-IR), Non-Isothermal Crystallization DSC Analysis, X-ray diffraction (XRD), Thermal gravimetric analysis (TGA), scanning electron microscope (SEM), mechanical and electrical performance testing of the samples were similar to our preliminary theses [16,17]. Besides, the melt flow index of samples was tested by a SANS ZRZ1452 melt flow indexer (MFI) (China), the testing temperature was $166^{\circ} \mathrm{C}$ and the loading weight was $2.16 \mathrm{~kg}$. The tensile performance of the samples was measured with a 4302 material testing machine from Instron Co., (USA), according to ISO 527-1993. The tensile test speed was $10 \mathrm{~mm} / \mathrm{min}$, and the sample length between bench 
marks was $25 \pm 0.5 \mathrm{~mm}$. The notched impact strength of the samples was measured with ZBC-4B impact testing machine from Xinsansi Co. (Shenzhen,China) according to ISO 179-1993 standards. The electrical conductivity of the samples was measured with ZC36 high insulation resistance meter spectrometer (China). The thermal conductivity of the samples was calculated from the thermal diffusivity which was measured by using a NETZSCH-LFA 447 instrument.
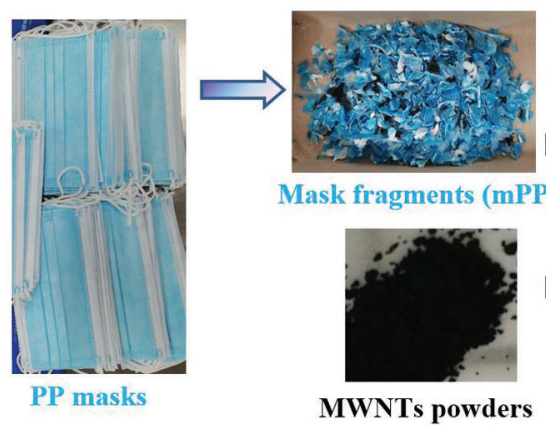

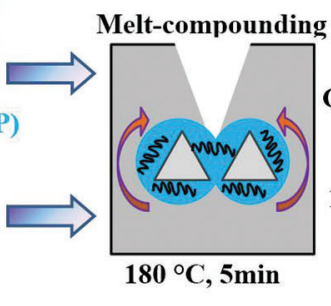

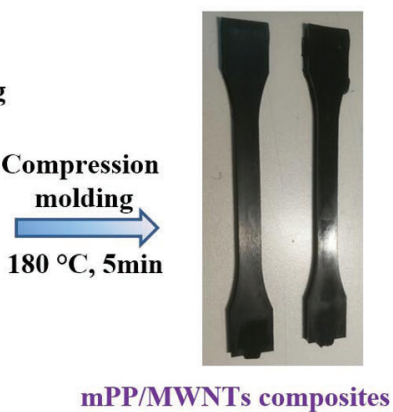

mPP/MWNTs composites

Figure 2: Preparation of mPP/MWNTs composites

\section{Results and Discussion}

The influence of the recycling of the PP due to chain scission could be deduced from the melt flow index (MFI) of the samples, as shown in Fig. 3. The primitive face mask in this paper denotes the sample of disposable face masks determining MFI after been cut into small pieces with scissors directly, without been compressed at $180^{\circ} \mathrm{C}$ for $5 \mathrm{~min}$ and internal mixed at $180^{\circ} \mathrm{C}$ for $5 \mathrm{~min}$ again. Through the comparison of primitive face mask and MPP in MFI determination, we can see the influence of hot pressing and internal mixing on the fluidity of polymer. When primitive face masks were processed into $\mathrm{mPP}$, the change of MFI was not obvious due to the low speed $(50 \mathrm{rpm})$ of melt processing in the torque rheometer. Therefore, chain scission did not occur in this processing. However, the MFI of the composites Rapidly decreased with MWNTs loading, indicating that the MWNTs fillers with large aspect ratio would reduce the melt fluidity of composites.

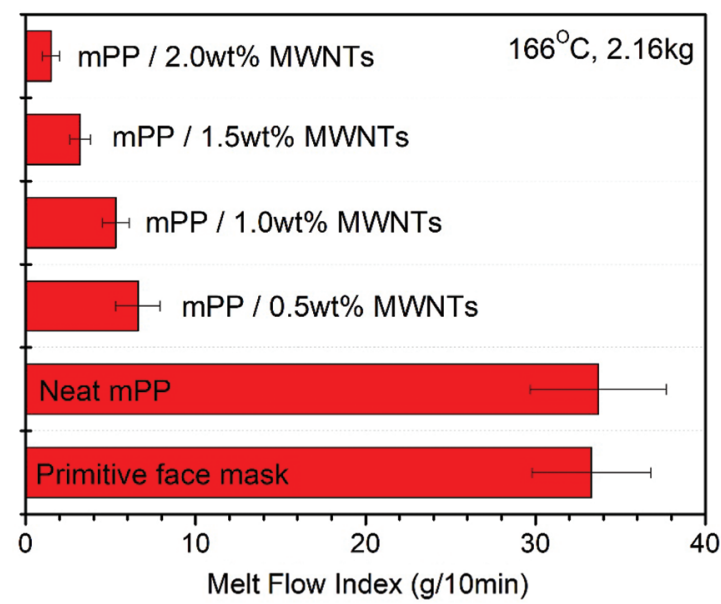

Figure 3: $\mathrm{MFI}$ of neat $\mathrm{mPP}$ and $\mathrm{mPP} / \mathrm{MWNTs}$ composites 
Fig. 4 displays the FT-IR curves of Neat mPP and mPP/MWNTs composites. Neat mPP exhibits typical peaks at $1458 \mathrm{~cm}^{-1}\left(-\mathrm{CH}_{3}\right.$ asymmetric deformation vibration), $1374 \mathrm{~cm}^{-1}\left(-\mathrm{CH}_{3}\right.$ symmetric deformation vibration). And the peaks at $1152 \mathrm{~cm}^{-1}$ (C-C stretching vibration in propylene chains), $973 \mathrm{~cm}^{-1}\left(-\mathrm{CH}_{3}\right.$ symmetric rocking vibration) demonstrate the head to tail structure in $\mathrm{mPP}$ chains [18]. For mPP/MWNTs composites, caused by the shielding effect of carbon hexatomic ring of MWNTs in infrared band, the peak intensity ascribed to mPP are weakened as the MWNTs loading [19]. Furthermore, there was no absorption peak of carbonyl group at $1716 \mathrm{~cm}^{-1}$ in the infrared spectra of all samples, which indicated that the samples did not undergo thermal oxidative degradation during the processing of this study.

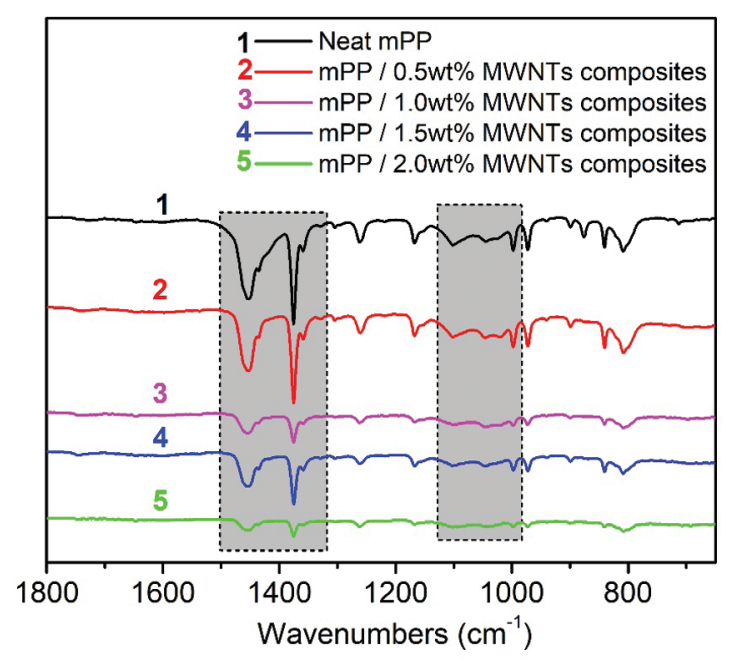

Figure 4: FT-IR curves of neat $\mathrm{mPP}$ and $\mathrm{mPP} / \mathrm{MWNTs}$ composites

The thermal stability of Neat $\mathrm{mPP}$ and $\mathrm{mPP} / \mathrm{MWNTs}$ composites are listed in Fig. 5a. For Neat mPP, the degradation stage during $380-450^{\circ} \mathrm{C}$ was due to fracture of C-C bond in $\mathrm{mPP}$ chains. The thermal stability of $\mathrm{mPP} / \mathrm{MWNTs}$ composites are enhanced in the same degradation stage, and the ultimate weight loss of mPP/MWNTs composites was fewer compared with Neat mPP. As shown in Fig. 5b, the corresponding DTG results obtained shown that the initial decomposing temperature and maximum decomposing temperature of $\mathrm{mPP}$ are postponed and the exothermic rate is retarded with adding the thermal stabilizer of MWNTs. The enhanced thermal stability of the composites come from the good thermal stability of the MWNTs their selves. Besides, good thermal conductivity of MWNTs can transfer the heat needed by the thermal decomposition of the composite in time.

The non-isothermal DSC results such as crystallization temperature $\left(\mathrm{T}_{\mathrm{c}}\right)$, the melting temperature $\left(\mathrm{T}_{\mathrm{m}}\right)$, the half peak width $(\Delta \mathrm{W})$, and the crystallinity $\left(\mathrm{X}_{\mathrm{c}}, \mathrm{DSC}\right)$ of Neat $\mathrm{mPP}$ and $\mathrm{mPP} / \mathrm{MWNTs}$ composites could be deduced from Figs. 6a and 6b, and were organized in Tab. 1 [20].

The change of $\mathrm{T}_{\mathrm{m}}$ and $\Delta \mathrm{W}$ of the composites was slight; however, $\mathrm{T}_{\mathrm{c}}$ of the composites improves with the MWNTs loading. The super cooling degree $\left(T_{m}-T_{c}\right)$ continues declining. The crystallinity $\left(X_{c}\right)$ of the composites presents the same tendency with $\mathrm{T}_{c}$, indicating the nucleation effect of MWNTs on $\mathrm{mPP}$ as heterogeneous nucleating agents.

XRD results were placed in Fig. 7. Neat mPP indicated summits of crystalline form $\alpha$ at $2 \theta=14.4^{\circ}$, $17.3^{\circ}, 19.2^{\circ}, 22.4^{\circ}$ and $30.1^{\circ}$, corresponding to the (110), (040), (130), (131), (041) and (111) planes, respectively. $\mathrm{mPP} / \mathrm{MWNT}$ composites shows different diffraction peaks at $2 \theta=26.1^{\circ}$ corresponding to (002) plane of carbon hexatomic ring, indicating the successful introduction of MWNTs in the matrix. 
Besides, the introduction of MWNTs promotes the growth of mPP crystals along (110) and (040) planes, resulting from the heterogeneous nucleation effect of MWNTs from crystal lattice in the composites [21].

The degree of crystallinity $\left(X_{c, X R D}\right)$ and crystallized grain sizes $\left(L_{h l k}\right)$ could be obtained via:

$X c, X R D=(I c /(I c+I a)) \times 100 \%$

where $I_{c}$ was the area contained in crystallization lines while $I_{a}$ was the area contained in amorphous lines.
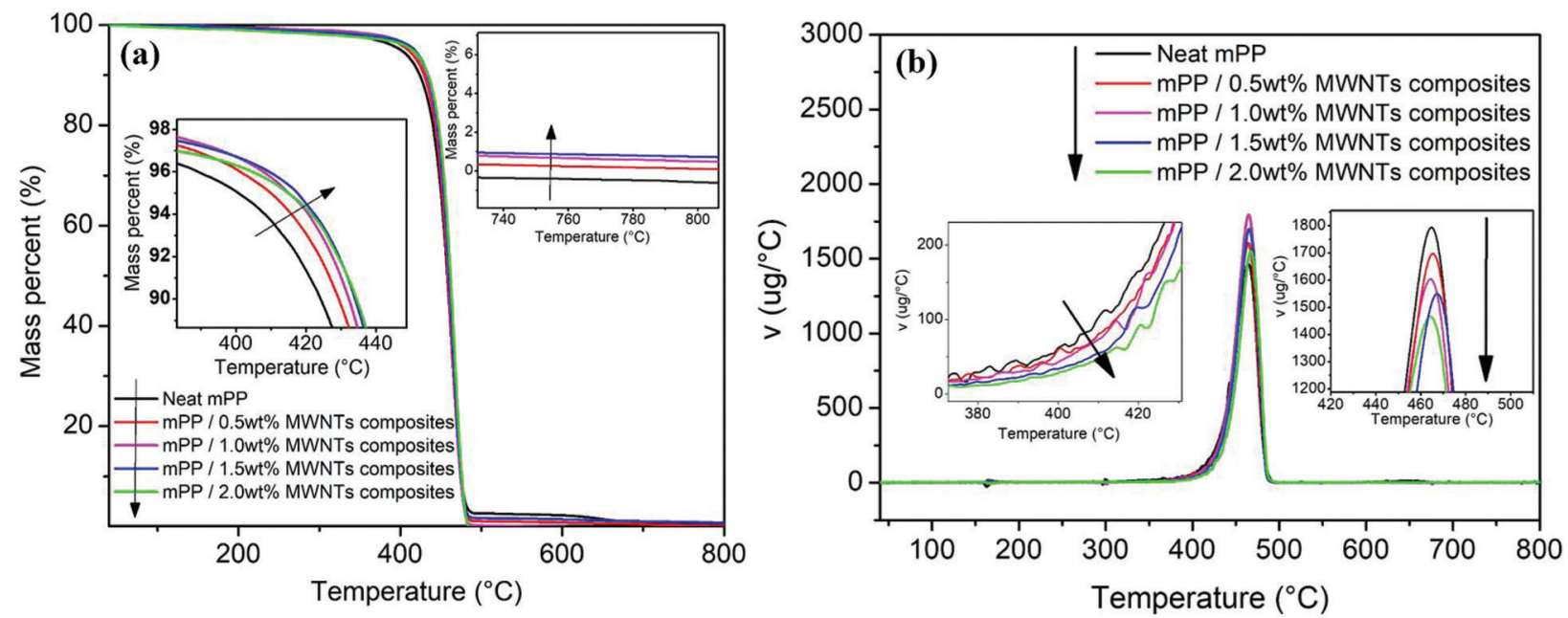

Figure 5: TG (a) and DTG (b) curves of neat mPP and mPP/MWNTs composites
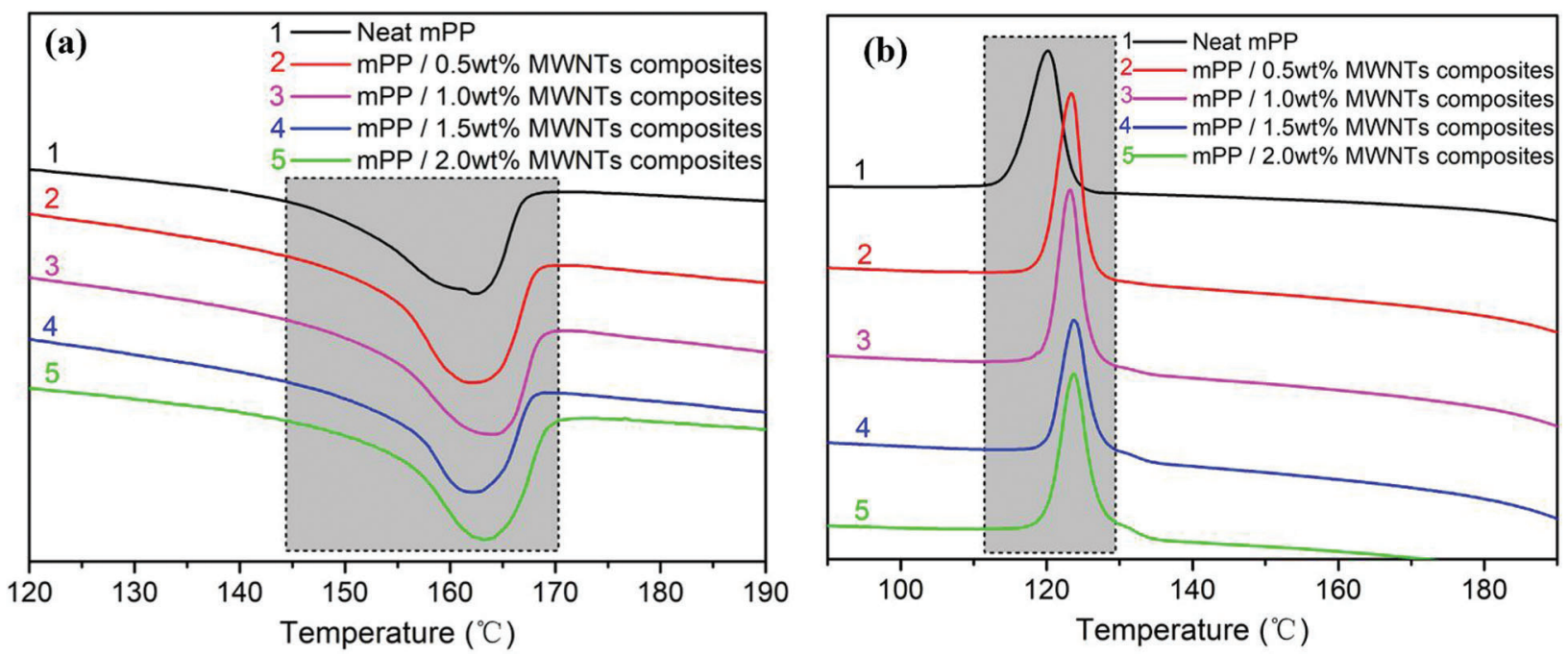

Figure 6: Non-isothermal DSC curves of mPP/MWNTs composites 
Formula of Scherrer

$L_{h l k}=k \lambda /(\beta \cos \theta)$

where $L_{h l k}$ was the size of crystalline grains in normal direction from $h l k$ planes; $k$ was constant of Scherrer $(k=0.89) ; \lambda$ was length of diffraction wave, about $0.154 \mathrm{~nm}$ while $\beta$ was full width of half maximum of the diffraction peak $(h l k) ; \theta$ was angle of Bragg.

For results in Tab. 1, the crystallinity of the composites kept improving with MWNTs loading, being consistent with non-isothermal DSC results, however, the crystalline grains size of the composites shows opposite rule of change. MWNTs acted the role of promoting the crystallisation of $\mathrm{mPP}$ by expediting the crystalline nucleation, leading to the improvement of amount for crystalline nucleus. Thus, grain size turns smaller by MWNTs loading.

Table 1: Non-isothermal DSC and XRD parameters of mPP/MWNTs composites with varying MWNTs content

\begin{tabular}{llllllll}
\hline $\begin{array}{l}\text { MWNTs } \\
\text { content }(\mathrm{wt} \%)\end{array}$ & $\begin{array}{l}\mathrm{T}_{\mathrm{m}} \\
\left({ }^{\circ} \mathrm{C}\right)\end{array}$ & $\begin{array}{l}\mathrm{T}_{\mathrm{m}}-\mathrm{T}_{\mathrm{c}} \\
\left({ }^{\circ} \mathrm{C}\right)\end{array}$ & $\begin{array}{l}\mathrm{T}_{\mathrm{c}} \\
\left({ }^{\circ} \mathrm{C}\right)\end{array}$ & $\begin{array}{l}\Delta \mathrm{W} \\
\left({ }^{\circ} \mathrm{C}\right)\end{array}$ & $\begin{array}{l}\mathrm{X}_{\mathrm{c}, \text { DSC }} \\
(\%)\end{array}$ & $\begin{array}{l}X_{c, X R D} \\
(\%)\end{array}$ & $\begin{array}{l}\text { Grain size in }(040) \\
\text { plane }(\mathrm{nm})\end{array}$ \\
\hline 0 & 161.5 & 41.4 & 120.1 & 8.6 & 42.6 & 51.2 & 14.3 \\
0.5 & 162.5 & 38.7 & 123.8 & 6.5 & 47.0 & 58.3 & 12.0 \\
1.0 & 162.9 & 38.8 & 124.1 & 6.7 & 48.4 & 66.7 & 11.3 \\
1.5 & 164.7 & 40.0 & 124.7 & 6.2 & 50.1 & 69.0 & 10.4 \\
2.0 & 164.1 & 39.2 & 124.9 & 6.5 & 51.7 & 73.2 & 9.5 \\
\hline
\end{tabular}

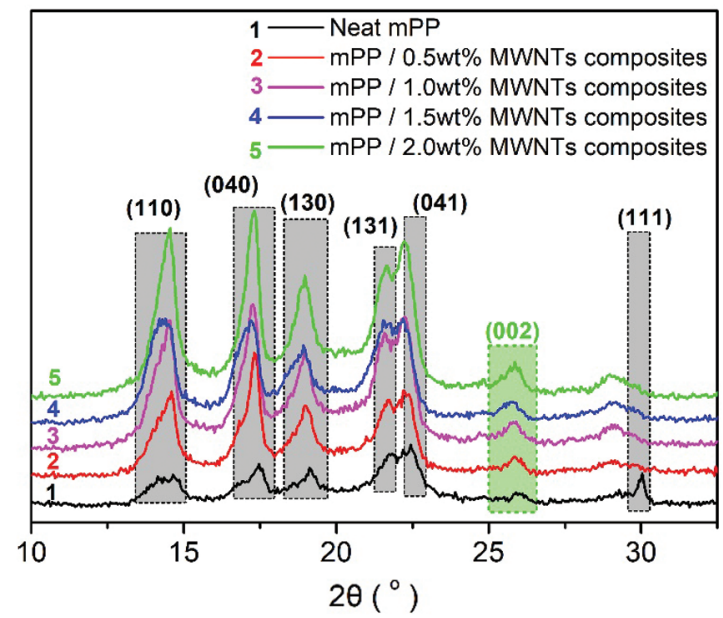

Figure 7: XRD curves of mPP/MWNTs composites

In Fig. 8a, the stress-strain curves of all samples showed a brittle fracture property without any yield points. The strength of $\mathrm{mPP} / \mathrm{MWNTs}$ composites enhance with increasing of MWNTs contents. The areas contained in stress-strain lines improved drastically compared to neat mPP, denoting the improving toughness of $\mathrm{mPP} / \mathrm{MWNTs}$ composites. Figs. $8 \mathrm{~b}-8 \mathrm{~d}$ tell the mechanical properties of $\mathrm{mPP} / \mathrm{MWNTs}$ 
composites with various MWNTs loading. The tensile strength of the composites increase gradually as MWNTs loading, reaching the maximum of $34.03 \mathrm{MPa}$ (increased by $809 \%$ ) at $2.0 \mathrm{wt} \% \mathrm{MWNTs}$, which was higher than common commercial grade polypropylene (such as $30.5 \mathrm{MPa}$ of T30S). The elongation at break of the composites also presented similar rule with impact strength, also meaning the toughening effects of cellulose skeleton of MWNTs on mPP matrix. The Young's modulus of the composites increased slowly and gradually stabilized with MWNTs loading. Caused by the high aspect ratio and high rigidity of MWNTs themselves, the ability of the composite to resist elastic deformation is enhanced. To sum up, our research shows a certain practical value in terms of mechanical enhancement.
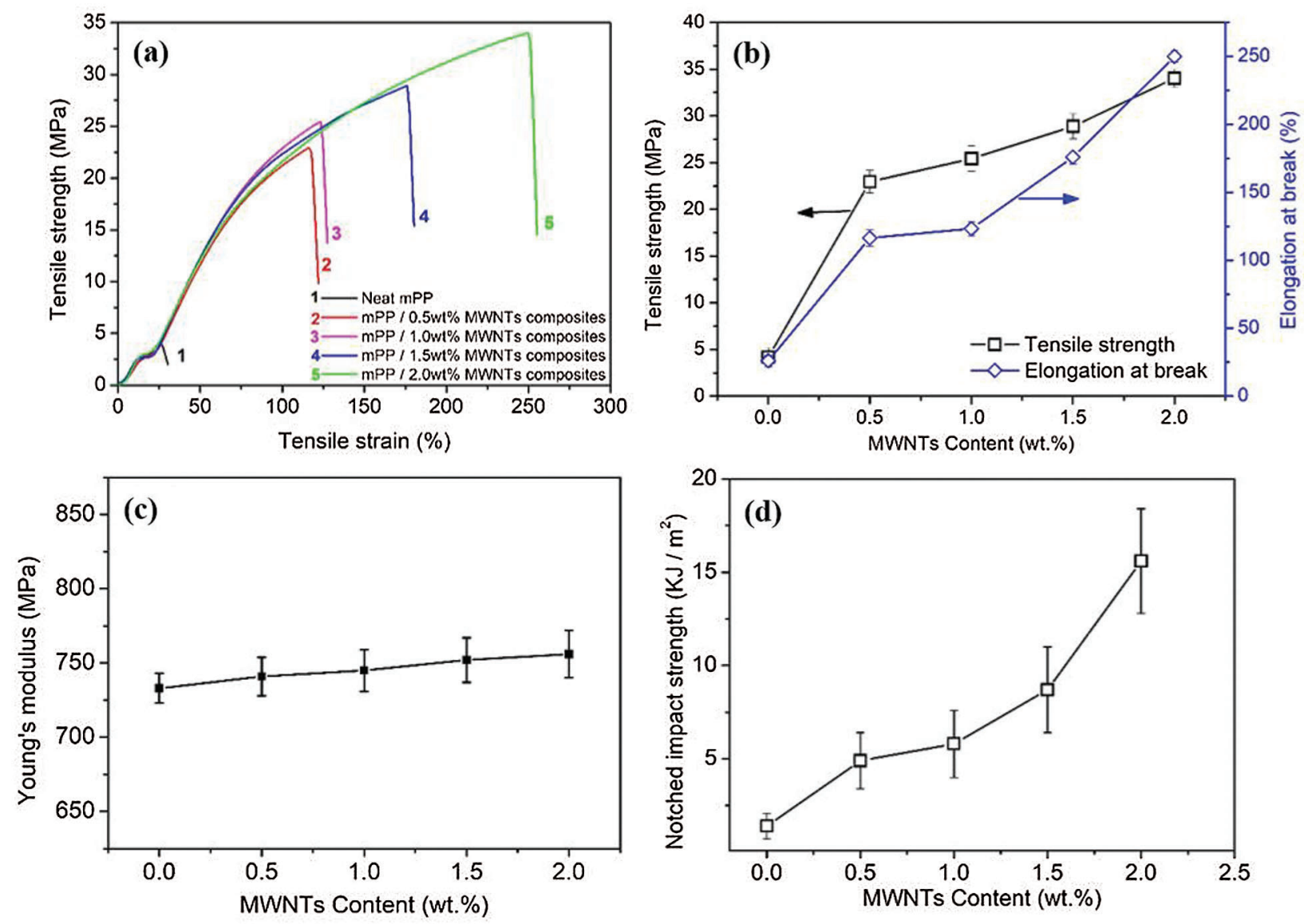

Figure 8: Mechanical properties $(\mathrm{a}-\mathrm{d})$ of neat $\mathrm{mPP}$ and $\mathrm{mPP} / \mathrm{MWNTs}$ composites

Fig. 9a shows electrical conductivity of the composites by different MWNTs content. The conductivity of Neat $\mathrm{mPP}$ was $1.6 \times 10^{-15} \mathrm{~S} / \mathrm{m}$. However, the conductivity in $\mathrm{mPP} / \mathrm{MWNTs}$ composites improves by MWNTs contents, while the percolation threshold was under $1.0 \mathrm{wt} \%$. By MWNTs contents of $2.0 \mathrm{wt} \%$, composite owns conductivity of $2.7 \times 10^{-8} \mathrm{~S} / \mathrm{m}$, suggesting the final formation of the conducting network in mPP matrix. The thermal conductivity of composites improves by MWNTs loading (Fig. 9b). The thermal conductivity of the composites containing $2.0 \mathrm{wt} \%$ MWNTs improves by $33 \%$. Herein, MWNTs were dispersed uniformly in highly fluid mPP matrix, leading to the available thermal energy transfer alongside the conducting network. 

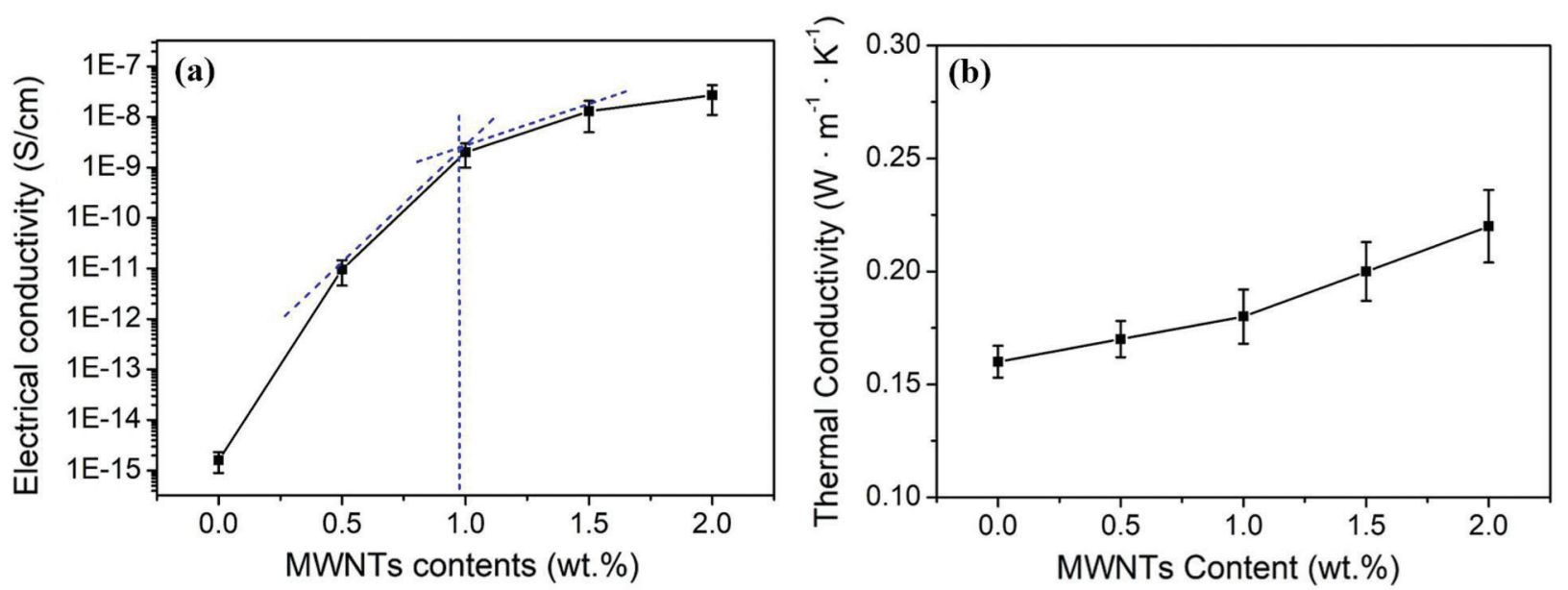

Figure 9: Electrical properties (a) and thermal properties (b) of neat mPP and mPP/MWNTs composites

When observed by the SEM of the fractures of the samples made at low magnification, as shown in Fig. 10a, there is no defect in the Neat mPP, demonstrating the correct preparation of the polymer composition. In Fig. 10b, the tensile fracture surface of $\mathrm{mPP} / 2.0 \mathrm{wt} \% \mathrm{MWNTs}$ composites was observed with SEM. It could be observed that MWNTs were uniformly dispersed in PP matrix. Firstly, just as the graphene, MWNTs were composed of hexagonal carbon atoms with low polarity, which has good compatibility with PP owning similarly low polarity [22]. Secondly, the recycled mPP from disposable face masks has a relatively small molecular weight, leading to the lower melt viscosity in the preparation of the composites, in which the MWNTs can be easier to uniformly dispersed in recycled PP matrix.
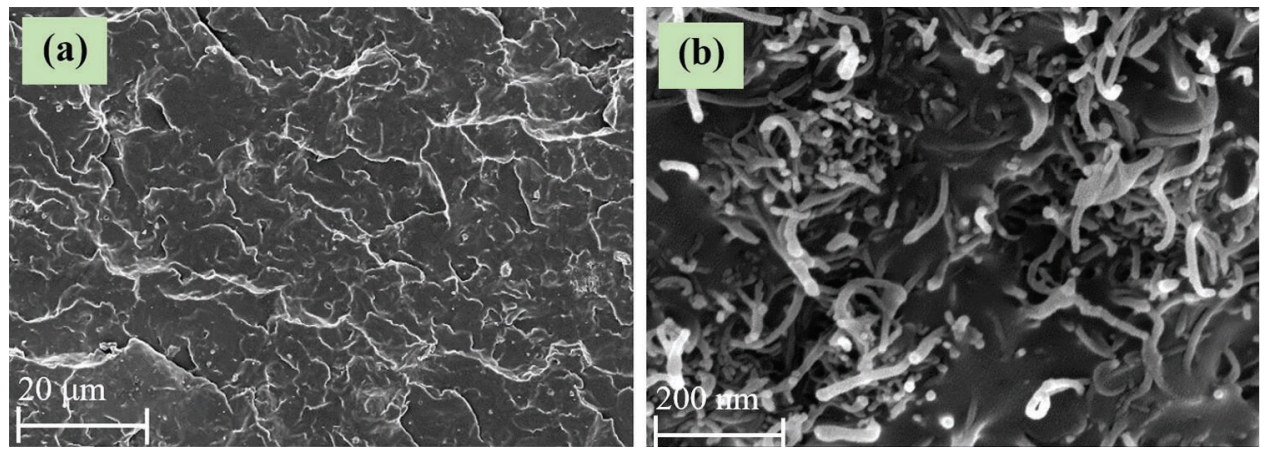

Figure 10: SEM image of tensile fracture surface of neat $\mathrm{mPP}(\mathrm{a})$ and $\mathrm{mPP} / 2.0 \mathrm{wt} \% \mathrm{MWNTs}$ composites (b)

Besides, the outer wall of pulled out MWNTs from mPP matrix is rough, attached with a large amount of $\mathrm{mPP}$ fragments, suggesting that there is a strong interfacial interaction between $\mathrm{mPP}$ and MWNTs. Moreover, MWNTs fibers interpenetrate with each other in mPP matrix to form conducting network. Thus, more conducting paths were constructed to transport carriers, and $\mathrm{mPP}$ matrix is locked in the conducting network to avoid rupture under strong external force, coincide with the mechanical and conducting properties.

\section{Conclusions}

Conductive polymer composites are fabricated by simple melt blending of mPP and MWNTs. The mechanical and electrical enhancement effect of the composites were investigated. In FT-IR analysis, 
caused by the shielding effect of carbon hexatomic ring of MWNTs in infrared band, the peak intensity ascribed to MPP are weakened as the MWNTs loading. TG denotes that the enhanced thermal stability of the composites results from MWNTs themselves. DSC and XRD indicated that MWNTs acted the role of promoting the crystallisation of $\mathrm{mPP}$ by expediting the crystalline nucleation, leading to the improvement of amount for crystalline nucleus. The tensile strength of the composites increase gradually as MWNTs loading, reaching the maximum of $34.03 \mathrm{MPa}$ (increased by $809 \%$ ) at $2.0 \mathrm{wt} \%$ MWNTs, which was higher than common commercial grade polypropylene (such as $30.5 \mathrm{MPa}$ of T30S). MWNTs fibers interpenetrate with each other in mPP matrix to form conducting network. Thus, more conducting paths were constructed to transport carriers, and $\mathrm{mPP}$ matrix is locked in the conducting network to avoid rupture under strong external force, leading to the improved mechanical, electrical and thermal properties of the composites.

Funding Statement: M. Xiang and S. Dong wishes to thank the National Natural Science Foundation of China (21908086 and 51801083), Changzhou Sci \& Tech Program (CJ20190035), Jiangsu Higher Education Institutions in China (19KJB610011) and Natural Science Foundation of Jiangsu Province (BK20181044).

Conflicts of Interest: The authors declare that they have no conflicts of interest to report regarding the present study.

\section{References}

1. Abboah-Offei, M., Salifu, Y., Adewale, B., Bayuo, J., Ofosu-Poku, R. et al. (2021). A rapid review of the use of face mask in preventing the spread of COVID-19. International Journal of Nursing Studies Advances, 3, 100013. DOI 10.1016/j.jijnsa.2020.100013.

2. Anastopoulos, I., Pashalidis, I. (2021). Single-use surgical face masks, as a potential source of microplastics: Do they act as pollutant carriers? Journal of Molecular Liquids, 326(3), 115247. DOI 10.1016/j.molliq.2020.115247.

3. Wang, M., Tang, X. H., Cai, J. H., Wu, H., Shen, J. B. et al. (2021). Fabrication, mechanisms and perspectives of conductive polymer composites with multiple interfaces for electromagnetic interference shielding: A review. Carbon, 177, 377-402. DOI 10.1016/j.carbon.2021.02.047.

4. Tang, L. P., Yang, S., Liu, D., Wang, C., Ge, Y. Q. et al. (2020). Two-dimensional porous coordination polymers and nano-composites for electrocatalysis and electrically conductive applications. Journal of Materials Chemistry A, 8(29), 14356-14383. DOI 10.1039/D0TA03356A.

5. Gao, J. F., Wang, L., Guo, Z., Li, B., Wang, H. et al. (2020). Flexible, superhydrophobic, and electrically conductive polymer nanofiber composite for multifunctional sensing applications. Chemical Engineering Journal, 381, 12. DOI 10.1016/j.cej.2019.122778.

6. Stanciu, N. V., Stan, F., Sandu, I. L., Fetecau, C., Turcanu, A. M. (2021). Thermal, Rheological, mechanical, and electrical properties of polypropylene/multi-walled carbon nanotube nanocomposites. Polymers, 13(2), 22. DOI 10.3390/polym13020187.

7. Belviso, C., Montano, P., Lettino, A., Toschi, F., Guarnaccio, A. (2021). Determining the role of the method used to recycle polypropylene waste materials from automotive industry using sepiolite and zeolite fillers. Journal of Material Cycles and Waste Management, 23(3), 965-975. DOI 10.1007/s10163-021-01184-w.

8. Ajorloo, M., Ghodrat, M., Kang, W. H. (2021). Incorporation of recycled polypropylene and fly ash in polypropylene-based composites for automotive applications. Journal of Polymers and the Environment, 29(4), 1298-1309. DOI 10.1007/s10924-020-01961-y.

9. Bahri, M. A. S., Ratnam, M. M., Khalil, H. (2020). Functionally graded wood filler-recycled polypropylene composite: Effect of mechanical loading on deflection of cantilever beam. Advanced Composites Letters, 29(2), 9. DOI 10.1177/2633366X20922856.

10. Barczewski, M., Andrzejewski, J., Majchrowski, R., Dobrzycki, K., Formela, K. (2021). Mechanical properties, microstructure and surface quality of polypropylene green composites as a function of sunflower husk waste filler particle size and content. Journal of Renewable Materials, 9(5), 841-853. DOI 10.32604/jrm.2021.014490. 
JRM, 2022, vol.10, no.3

11. Battegazzore, D., Cravero, F., Frache, A. (2020). Is it possible to mechanical recycle the materials of the disposable filtering masks? Polymers, 12(11), 18. DOI 10.3390/polym12112726.

12. Wu, Z. G., Zheng, H. Y., Gu, J., Li, F., Lv, R. L. et al. (2020). Effects of different temperature and time durations of virus inactivation on results of real-time fluorescence PCR testing of COVID-19 viruses. Current Medical Science, 40(4), 614-617. DOI 10.1007/s11596-020-2224-y.

13. Ju, S., Yoon, J., Sung, D., Pyo, S. (2020). Mechanical properties of coal ash particle-reinforced recycled plasticbased composites for sustainable railway sleepers. Polymers, 12(10), 15. DOI 10.3390/polym12102287.

14. Carrete, I. A., Quinonez, P. A., Bermudez, D., Roberson, D. A. (2021). Incorporating textile-derived cellulose fibers for the strengthening of recycled polyethylene terephthalate for 3D printing feedstock materials. Journal of Polymers and the Environment, 29(2), 662-671. DOI 10.1007/s10924-020-01900-x.

15. Echeverria, C. A., Pahlevani, F., Sahajwalla, V. (2020). Valorisation of discarded nonwoven polypropylene as potential matrix-phase for thermoplastic-lignocellulose hybrid material engineered for building applications. Journal of Cleaner Production, 258(54), 11. DOI 10.1016/j.jclepro.2020.120730.

16. Xiang, M., Xu, S., Li, C. J., Ye, L. (2016). Monomer casting nylon-6-b-polyether amine copolymers: Synthesis and antistatic property. Polymer Engineering and Science, 56(7), 817-828. DOI 10.1002/pen.24310.

17. Xiang, M., Yang, R., Yang, J., Zhou, S., Zhou, J. et al. (2019). Fabrication of polyamide 6/reduced graphene oxide nano-composites by conductive cellulose skeleton structure and its conductive behavior. Composites Part B: Engineering, 167, 533-543. DOI 10.1016/j.compositesb.2019.03.033.

18. Gopanna, A., Mandapati, R. N., Thomas, S. P., Rajan, K., Chavali, M. (2019). Fourier transform infrared spectroscopy (FTIR), Raman spectroscopy and wide-angle X-ray scattering (WAXS) of polypropylene (PP)/ cyclic olefin copolymer (COC) blends for qualitative and quantitative analysis. Polymer Bulletin, 76(8), 42594274. DOI 10.1007/s00289-018-2599-0.

19. Wang, P. H., Ghoshal, S., Gulgunje, P., Verghese, N., Kumar, S. (2016). Polypropylene nanocomposites with polymer coated multiwall carbon nanotubes. Polymer, 100(2), 244-258. DOI 10.1016/j.polymer.2016.07.070.

20. Schawe, J. E. K. (2015). Analysis of non-isothermal crystallization during cooling and reorganization during heating of isotactic polypropylene by fast scanning DSC. Thermochimica Acta, 603, 85-93. DOI 10.1016/j. tca.2014.11.006.

21. Parija, S., Bhattacharyya, A. R. (2017). Multiwalled carbon nanotubes-based polypropylene composites: Influence of interfacial interaction on the crystallization behavior of polypropylene. Polymer Engineering and Science, 57(2), 183-196. DOI 10.1002/pen.24399.

22. Wang, H. Q., Li, Z. W., Hong, K. L., Chen, M. N., Qiao, Z. et al. (2019). Property improvement of multi-walled carbon nanotubes/polypropylene composites with high filler loading via interfacial modification. RSC Advances, 9(50), 29087-29096. DOI 10.1039/C9RA05493F. 